\title{
Combining Particle MCMC with Rao-Blackwellized Monte Carlo Data Association for Parameter Estimation in Multiple Target Tracking
}

\author{
Juho Kokkala*, Simo Särkkä \\ Aalto University \\ Espoo, Finland
}

\begin{abstract}
We consider state and parameter estimation in multiple target tracking problems with data association uncertainties and unknown number of targets. We show how the problem can be recast into a conditionally linear Gaussian state-space model with unknown parameters and present an algorithm for computationally efficient inference on the resulting model. The proposed algorithm is based on combining the Rao-Blackwellized Monte Carlo data association algorithm with particle Markov chain Monte Carlo algorithms to jointly estimate both parameters and data associations. Both particle marginal Metropolis-Hastings and particle Gibbs variants of particle MCMC are considered. We demonstrate the performance of the method both using simulated data and in a real-data case study of using multiple target tracking to estimate the brown bear population in Finland.
\end{abstract}

\footnotetext{
* Corresponding author

Email addresses: juho.kokkala@aalto.fi (Juho Kokkala), simo.sarkka@aalto.fi (Simo Särkkä)

URL: http://becs.aalto.fi/ jkokkala (Juho Kokkala), http://becs.aalto.fi/ ssarkka (Simo Särkkä)
} 
Keywords: Multiple target tracking, Rao-Blackwellized Monte Carlo data association, Particle filtering, Sequential Monte Carlo, Particle MCMC, Parameter estimation

\section{Introduction}

This paper is concerned with multiple target tracking (MTT), that is, with the problem of estimating the locations or states of several moving objects (targets) based on noisy measurements (see, e.g., Blackman and Popoli, 1999; Bar-Shalom et al., 2001; Kirubarajan and Bar-Shalom, 2005; Mahler, 2007b; Challa et al., 2011). The challenge in MTT is that in addition to estimating the locations, one needs to solve the subproblems of estimating the number of targets and determining which target each measurement comes from, known as the data association problem. MTT methods have been applied, for example, to aircraft tracking (Hwang et al., 2004), video

surveillance (Rao and Satyanarayana, 2013), evolutionary clustering (Mestre and Fitzgerald, 2013), and estimating the size of animal population (Abbas, 2011). In this paper we formulate the multiple target tracking problem as a Rao-Blackwellized particle filtering problem following Särkkä et al. (2007) and then show how we can use state-of-the-art particle Markov chain Monte Carlo (PMCMC) methods (Andrieu et al., 2010) to estimate the parameters of the model.

In the Rao-Blackwellized Monte Carlo data association (RBMCDA) algorithm proposed by Särkkä et al. (2007), target movements and measurements given targets are assumed to follow a linear-Gaussian state-space model. Thus, conditional on the data associations, posterior distributions for the 
target locations are obtained in closed form using the Kalman filter Kalman, 1960). This enables the use of the Rao-Blackwellized particle filter (RBPF, Akashi and Kumamoto, 1977; Doucet et al., 2000b a; Chen and Liu, 2000; Särkkä, 2013) to sample the data associations. Vihola (2007) proposed a similar RBPF filter, where the conditional linear-Gaussian model is formulated in the random set framework.

In this paper we show how the RBMCDA algorithm of Särkkä et al. (2007) can be extended to joint estimation of unknown parameters along with the target states. In the Bayesian framework (Gelman et al., 2013; Särkkä, 2013), parameters are modeled as random variables and the goal of parameter estimation is to compute the posterior probability distributions over parameters conditional on observations. Markov chain Monte Carlo (MCMC) methods are typically used to produce samples from the posterior distributions. In the context of state-space models, such as tracking problems, one needs to jointly sample both from the posterior of the parameters and the posterior of the states. Particle MCMC (PMCMC) algorithms (Andrieu et al., 2010) are a special class of MCMC algorithms that use particle filter algorithms to produce samples of state variables within MCMC. In this paper, we propose combining the RBMCDA and PMCMC algorithms to sample from the joint posterior distribution of data associations and parameters. This combined algorithm is intended for models where the movement and measurements from individual targets follow a linear-Gaussian state-space model conditional on the fixed number of unknown parameters. However, it is also possible to treat approximately linear-Gaussian state-space models by replacing the Kalman filters with extended Kalman filters (EKF), unscented Kalman filters (UKF), 
or other non-linear filters (see, e.g., Särkkä, 2013).

Using PMCMC in MTT has been suggested previously by Vu et al. (2014) and Duckworth (2012). These approaches use MCMC to propose data associations and the particle filter to sample target states conditional on the data associations. The algorithm of $\mathrm{Vu}$ et al. (2014) does not sample static parameters at all, while Duckworth (2012) samples static parameters within the particle filter. Our proposed algorithm differs from these in that the MCMC is used to propose static parameters while data associations and the number of targets are sampled in the RBMCDA filter.

The use of PMCMC in combination with Rao-Blackwellized particle filters has been proposed before in other contexts (e.g. Chopin, 2010; Peters and Cornebise, 2010). However, the particular method proposed in this article is novel since the combination of PMCMC and Rao-Blackwellized particle filters, in particular RBMCDA, has, to our knowledge, not been used in the multiple target tracking context.

The remainder of the article is structured as follows. In Section 1.1, we present a brief survey of the multiple target tracking literature. In Section 2, we review the particle filtering and particle MCMC algorithms, and in Section 3 the RBMCDA algorithm. In Section 4, we present the combined RBMCDA-PMCMC algorithms. In the numeric experiments in Section 4 , we use simulated data to compare the performance of the particle Gibbs with varying numbers of particles. We also present a real-data application of the algorithm to estimating the bear population of Finland based on a database of field-signs and direct observations. Pseudocodes for the algorithms are presented in Appendix A. 


\subsection{Multiple Target Tracking Methods}

Various filtering approaches for multiple target tracking have been proposed in literature. Joint probabilistic data association (JPDA) (Fortmann et al., 1980) approximates the joint density by a Gaussian distribution. In the update step, the measurements are weighted by data association probabilities. In multiple hypothesis tracking (MHT) (Reid, 1979, Blackman, 2004), target state distributions are maintained for different data association histories. To prevent combinatorial explosion, heuristics are employed to discard unlikely hypotheses.

Multiple particle filtering (Bugallo et al., 2007; Djuric and Bugallo, 2009) is based on tracking each target with a separate particle filter and approximatively combining the information in the weight update. More recently, Closas and Bugallo (2012) proposed a refinement where the weight computation is iterated in a game-theory-inspired manner. Another approach based on partitioning the state is the Independent partition particle filter (Orton and Fitzgerald, 2002), where the state is partitioned so that states of clearly separate targets are sampled independently. Yi et al. (2013) provided a different view of the target independence approximation - they used the assumption to improve approximation of the filter predicted density rather than for independently propagating target states.

Random set based MTT approaches such as probability hypothesis density (PHD) (Mahler, 2003, 2007b) filtering are based on the theory of finite set statistics (FISST, Mahler, 2007b). The joint random set distribution is often approximated with the PHD, which a density whose integral gives the expected number of targets in the region. The PHD may further be approxi- 
mated by Gaussian mixtures (Vo and Ma, 2006) or particle filters (Vo et al. 2003). For the particle filter PHD approach, Clark and Bell (2007) proposed to assign the particles to target labels by expectation-maximization or kmeans clustering. Clark et al. (2007) proposed a particle PHD filter where the particles represent a mixture of Gaussians rather than point masses. In cardinalized PHD (Mahler, 2007a), the probability distribution over number of targets is propagated along the PHD. Multi-target multi-Bernoulli filtering (MeMBer) (Vo et al., 2009) is based on target-wise densities and independent existence probabilities. More recently, Ravindra et al. (2012) proposed a MeMBer filter where the independence of existence probabilities is preserved by modifying the posterior densities of targets while preserving the random finite set (RFS) density. A related idea is the set JPDA method (Svensson et al., 2011) where the posterior after JPDA update is modified to improve Gaussian mixture estimation while preserving the RFS density. Recently, Svensson and Morelande (2014) proposed formulating the multiple target tracking problem as computing posterior distributions over random finite sets of trajectories directly, rather than random finite sets of states.

The RBMCDA (Särkkä et al., 2007) algorithm used in this paper is based on assuming linear-Gaussian target dynamics and measurements and then using a Rao-Blackwellized particle filter, where Kalman filter is used to track target states and the particle approximation to approximate the distribution over data associations. A related idea by Vihola (2007) proposed a RBPF filter, where the conditional linear-Gaussian model is formulated in the random set framework. Petetin et al. (2014) used a Rao-Blackwellized particle filter within the PHD framework. 


\section{Particle Filtering and Particle MCMC}

Consider a state-space model (see, e.g., Särkkä, 2013) with measurements $\mathbf{y}_{1}, \ldots, \mathbf{y}_{T} \in \mathbb{R}^{m}$, hidden states $\mathbf{x}_{0}, \ldots, \mathbf{x}_{T} \in \mathbb{R}^{n}$, and parameters $\boldsymbol{\theta} \in \mathbb{R}^{d}$, which consists of the Markovian dynamic model

$$
\mathbf{x}_{k} \sim p\left(\mathbf{x}_{k} \mid \mathbf{x}_{k-1}, \boldsymbol{\theta}\right)
$$

and the measurement model

$$
\mathbf{y}_{k} \sim p\left(\mathbf{y}_{k} \mid \mathbf{x}_{k}, \boldsymbol{\theta}\right)
$$

When the parameters $\boldsymbol{\theta}$ are fixed, the state sequence $\mathbf{x}_{0: T}$ is assumed to be Markovian and the measurements are assumed to be conditionally independent given the states. In the following, we briefly review the particle filtering (sequential importance resampling, SIR) algorithm for approximating the filtering distributions of the states, that is, $p\left(\mathbf{x}_{k} \mid \mathbf{y}_{1: k}, \boldsymbol{\theta}\right)$ and the particle MCMC algorithms that combine particle filtering with MCMC to sample from the joint posterior of the parameters and the states, $p\left(\boldsymbol{\theta}, \mathbf{x}_{0: T} \mid \mathbf{y}_{1: T}\right)$.

\subsection{Particle filtering}

In sequential importance resampling type particle filtering (Doucet et al., $2000 \mathrm{~b})$, the filtering distribution at time step $k, p\left(\mathbf{x}_{k} \mid \mathbf{y}_{1: k}\right)$, is approximated by a finite set of $N$ discrete particles with weights, $\left\{\left(w_{k}^{(i)}, \tilde{\mathbf{x}}_{k}^{(i)}\right): i=1, \ldots, N\right\}$. This is interpreted as the density approximation

$$
p\left(\mathbf{x}_{k} \mid \mathbf{y}_{1: k}\right) \approx \sum_{i=1}^{N} w_{k}^{(i)} \delta\left(\mathbf{x}_{k}-\tilde{\mathbf{x}}_{k}^{(i)}\right),
$$

where $\delta(\cdot)$ is the Dirac delta function. The particle filtering algorithm iterates the following steps through the measurements $k=1, \ldots, T$ : 
1. Sample new particles from an importance distribution: $\tilde{\mathbf{x}}_{k}^{(i)} \sim \pi\left(\mathbf{x}_{k} \mid\right.$ $\left.\tilde{\mathbf{x}}_{k-1}^{(i)}, \mathbf{y}_{k}\right)$.

2. Compute updated weights: $v_{k}^{(i)}=w_{k-1}^{(i)} \frac{p\left(\tilde{\mathbf{x}}_{k}^{(i)}\right) p\left(\mathbf{y}_{k} \mid \tilde{\mathbf{x}}_{k}^{(i)}\right)}{\pi\left(\mathbf{x}_{k} \mid \tilde{\mathbf{x}}_{k-1}^{(i)}, \mathbf{y}_{k}\right)}$.

3. Normalize weights: $w_{k}^{(i)}=\frac{v_{k}^{(i)}}{\sum_{i} v_{k}^{(i)}}$.

4. Resample: if necessary, draw $N$ new particle values $\tilde{\mathbf{x}}_{k}^{(i)}$ from the original $\tilde{\mathbf{x}}_{k}^{(i)}$ with probabilities $w_{k}$.

The purpose of the resampling step is to avoid degeneracy where one particle attains all weight. It may be performed periodically with a fixed interval or adaptively based on effective sample size (Liu and Chen, 1995) declining below a threshold.

For purposes of parameter estimation, the particle filter can also be used to form an approximation to the marginal likelihood $p\left(\mathbf{y}_{1: T} \mid \boldsymbol{\theta}\right)$ (see, e.g., Andrieu et al., 2004; Särkkä, 2013):

$$
\hat{p}\left(\mathbf{y}_{1: T} \mid \boldsymbol{\theta}\right)=\prod_{k=1}^{T} \hat{p}\left(\mathbf{y}_{k} \mid \mathbf{y}_{1: k-1}, \boldsymbol{\theta}\right),
$$

where

$$
\hat{p}\left(\mathbf{y}_{k} \mid \mathbf{y}_{1: k-1}, \boldsymbol{\theta}\right)=\sum_{i=1}^{N} v_{k}^{(i)} .
$$

When combined with Markov chain Monte Carlo (MCMC), this leads to so called particle MCMC (PMCMC) methods (Andrieu et al., 2010).

\subsection{Rao-Blackwellized Particle Filter}

For models, where the filtering problem is analytically tractable conditional on some subset of variables, one may reduce the variance of the importance weights by the Rao-Blackwellized particle filter Akashi and Ku- 
mamoto, 1977; Doucet et al., 2000b a; Chen and Liu, 2000), where the particle filter is employed only for the non-analytically tractable subset, and the tractable part is marginalized analytically. For example, in conditionally linear-Gaussian models of the form

$$
\begin{aligned}
\mathbf{x}_{k} & \sim \mathcal{N}\left(\mathbf{A}_{k-1}\left(\mathbf{u}_{k-1}\right) \mathbf{x}_{k-1}, \mathbf{Q}_{k-1}\left(\mathbf{u}_{k-1}\right)\right) \\
\mathbf{y}_{k} & \sim \mathcal{N}\left(\mathbf{H}_{k}\left(\mathbf{u}_{k}\right) \mathbf{x}_{k}, \mathbf{R}_{k}\left(\mathbf{u}_{k}\right)\right) \\
\mathbf{u}_{k} & \sim p\left(\mathbf{u}_{k} \mid \mathbf{u}_{k-1}\right),
\end{aligned}
$$

the particles of the Rao-Blackwellized particle filter contain samples of the latent variables $\mathbf{u}_{k}$, and the states $\mathbf{x}_{k}$ are marginalized out using the Kalman filter. Although we usually assume that the latent variables are a priori Markovian, the algorithm generalizes without modification to the non-Markovian (but causal) case. That is, the last equation above may be generalized to $p\left(\mathbf{u}_{k} \mid \mathbf{u}_{1: k-1}\right)$.

\subsection{Particle $M C M C$}

The idea of using particle filters within a Markov chain Monte Carlo (MCMC) sampler was suggested by, for example, Fernández-Villaverde and Rubio-Ramírez (2007); Jones et al. (2010). Theoretical justification that these particle MCMC algorithms indeed produce Markov chains that converge to the joint posterior of the states and parameters was provided by Andrieu et al. (2010). In this section, we discuss two different particle MCMC algorithms, both introduced by Andrieu et al. (2010). First, we discuss particle marginal Metropolis-Hastings, which is based on the likelihood approximation produced by the particle filter. Second, we discuss particle Gibbs where a modification of the particle filter called conditional sequential Monte Carlo is used to move in the space of state sequences. 
The particle marginal Metropolis-Hastings (PMMH) algorithm is a variant of the Metropolis-Hastings algorithm, where the exact evaluation of the likelihood (and posterior) is replaced by running the particle filter and using the approximate likelihood. The algorithm is initialized by selecting initial parameters $\boldsymbol{\theta}^{0}$ and running the particle filter to obtain approximate marginal likelihood $\hat{p}\left(\boldsymbol{\theta} \mid \mathbf{y}_{1: T}\right)$. Then, the algorithm produces samples from the parameters and particle sets, $\left(\boldsymbol{\theta}^{1}, \mathbf{x}_{1: T}^{1,(1: N)}, w_{T}^{1,(1: N)}\right),\left(\boldsymbol{\theta}^{2}, \mathbf{x}_{1: T}^{2,(1: N)}, w_{T}^{2,(1: N)}\right), \ldots$ by iterating the following steps

1. Draw proposed parameters: $\boldsymbol{\theta}^{*} \sim q\left(\boldsymbol{\theta}^{*} \mid \boldsymbol{\theta}^{j-1}\right)$

2. Run the particle filter (Section 2.1) using the parameters $\boldsymbol{\theta}^{*}$ to obtain weighted set of particles $\left(w_{T}^{*,(1: N)}, \mathbf{x}_{1: T}^{*,(1: N)}\right)$ and a marginal likelihood estimate $\hat{p}\left(\mathbf{y}_{1: T} \mid \boldsymbol{\theta}^{*}\right)(\mathrm{Eq} \cdot 4)$

3. With probability

$$
\alpha_{j}=\min \left(1, \frac{q\left(\boldsymbol{\theta}^{j-1} \mid \boldsymbol{\theta}^{*}\right)}{q\left(\boldsymbol{\theta}^{*} \mid \boldsymbol{\theta}^{j-1}\right)} \frac{\hat{p}\left(\mathbf{y}_{1: T} \mid \boldsymbol{\theta}^{*}\right)}{\hat{p}\left(\mathbf{y}_{1: T} \mid \boldsymbol{\theta}^{j-1}\right)} \frac{p\left(\boldsymbol{\theta}^{*}\right)}{p\left(\boldsymbol{\theta}^{j-1}\right)}\right)
$$

accept the proposal, that is:

$$
\begin{aligned}
& \left(\boldsymbol{\theta}^{j}, w_{T}^{j,(1: N)}, \mathbf{x}_{1: T}^{j,(1: N)}, \hat{p}\left(\mathbf{y}_{1: T} \mid \boldsymbol{\theta}^{j}\right)\right) \\
:= & \left(\boldsymbol{\theta}^{*}, w_{T}^{*,(1: N)}, \mathbf{x}_{1: T}^{*,(1: N)}, \hat{p}\left(\mathbf{y}_{1: T} \mid \boldsymbol{\theta}^{*}\right)\right) .
\end{aligned}
$$

4. If the proposal is not accepted, copy the values from previous iteration:

$$
\begin{aligned}
& \left(\boldsymbol{\theta}^{j}, w_{T}^{j,(1: N)}, \mathbf{x}_{1: T}^{j,(1: N)}, \hat{p}\left(\mathbf{y}_{1: T} \mid \boldsymbol{\theta}^{j}\right)\right) \\
:= & \left(\boldsymbol{\theta}^{j-1}, w_{T}^{j-1,(1: N)}, \mathbf{x}_{1: T}^{j-1,(1: N)}, \hat{p}\left(\mathbf{y}_{1: T} \mid \boldsymbol{\theta}^{j-1}\right)\right) .
\end{aligned}
$$

Samples from the state, $\mathbf{x}_{1: T}^{j}$, may be obtained by drawing one particle from the accepted particles $\mathbf{x}_{1: T}^{j,(1: N)}$ with using the importance weights $w_{T}^{j,(1: N)}$ 
as probabilities. The Markov chain produced by the PMMH algorithm is ergodic in an extended space consisting of the parameters and the particle sets so that the marginal stationary distribution in the states-and-parameters space is the correct posterior distribution (Andrieu et al. 2010). The particle Metropolis-Hastings algorithm may also be interpreted as a Multiple Try Metropolis algorithm, as Martino et al. (2015) point out.

The particle Gibbs algorithm is an MCMC algorithm moving in the joint space of $\left(\boldsymbol{\theta}, \mathbf{x}_{1: T}\right)$. The particle Gibbs uses a regular MCMC, namely Gibbs sampling, step to draw new parameter values conditional on the states and a variant of particle filter, conditional SMC, to sample new states. The conditional SMC is a variant of the particle filter that takes the current state sequence as input and fixes the states for one particle to the input sequence instead of drawing them from the importance distributions. That is, instead of drawing $\mathbf{x}_{k}^{(1)}$ from the importance distribution $q\left(\mathbf{x}_{k} \mid \mathbf{x}_{k-1}^{(1)}, \mathbf{y}_{k}\right)$, the value of $\mathbf{x}_{k}^{(1)}$ is set to the old value of $\mathbf{x}_{k}$. For particles $2, \ldots, N$ the algorithm proceeds exactly as the particle filter. Note that the weights are nevertheless recomputed even for the fixed particle as if the states were sampled from the importance distribution. After running the CSMC, $\mathbf{x}_{1: T}^{j}$ is sampled among the particles using the importance weights. In total, the particle Gibbs algorithm iterates the following steps:

1. Draw $\boldsymbol{\theta}^{j} \sim p\left(\boldsymbol{\theta} \mid \mathbf{x}_{1: T}^{j-1}\right)$

2. Generate $\left(\mathbf{x}_{1: T}^{j,(1: N)}, w_{1: T}^{j,(1: N)}\right)$ by running the conditional SMC using parameters $\boldsymbol{\theta}^{j}$ and fixing the first particle to $\mathbf{x}_{1: T}^{j-1}$.

3. Draw $\mathbf{x}_{1: T}^{j}$ from $\mathbf{x}_{1: T}^{j,(1: N)}$ with probabilities $w_{T}^{j,(1: N)}$.

Since the joint posterior distribution $p\left(\mathbf{x}_{0: T}, \boldsymbol{\theta} \mid \mathbf{y}_{1: T}\right)$ is an invariant dis- 
tribution for both the CSMC move and the parameter sampling move, the resulting particle Gibbs algorithm is a MCMC sampler targeting the joint posterior distribution (Andrieu et al. 2010).

Andrieu et al. (2010) also show that it is possible to improve the MCMC estimates by using the state sequences produced by all particles rather than only one state sequence selected per MCMC step. In particle Gibbs, all particles may be taken as samples weighted by their respective importance weights. Furthermore, in PMMH one may also use the particles corresponding to rejected parameter proposals by weighting the new particle set and the particle set corresponding to the last accepted proposal by the MetropolisHastings acceptance probability.

Combining Rao-Blackwellized particle filters with PMCMC was already suggested by Chopin (2010) and Peters and Cornebise (2010). Naturally, since the RBPF is a particle filter in the state space of the latent variables $\mathbf{u}$, using it in a PMCMC algorithm produces a MCMC sampler targeting the

joint posterior $p\left(\mathbf{u}_{0: T}, \boldsymbol{\theta} \mid \mathbf{y}_{1: T}\right)$. Whiteley et al. (2010) combined the discrete particle filter (Fearnhead and Clifford, 2003) with PMCMC to do inference in switching state-space models. In addition, Rao-Blackwellized PMCMC has been used by Nevat et al. (2011) in channel tracking in wireless relay networks, by Minvielle et al. (2014) in an electromagnetic inverse problem, and by Peters et al. (2013) in the context of a financial commodity model.

\section{Rao-Blackwellized Monte Carlo Data Association}

In this section, we review the RBMCDA algorithm proposed by Särkkä et al. (2007). The algorithm is formulated for models where the target dy- 
namics are linear with Gaussian process noise, and the measurements conditional on data associations are a linear function of target states plus Gaussian measurement noise. However, as was shown in Särkkä et al. (2007), it is also possible to handle non-linear state-space models by replacing the Kalman filters in the algorithm non-linear extensions such as extended Kalman filters (EKF), unscented Kalman filters (UKF), or more general non-linear Gaussian filters Särkkä (2013).

We denote the state of the $j$ th target at $k$ th time step by $\mathbf{x}_{k, j}$. The dynamics are assumed to be linear with Gaussian noise, that is,

$$
p\left(\mathbf{x}_{k, j} \mid \mathbf{x}_{k-1, j}\right)=\mathcal{N}\left(\mathbf{x}_{k, j} \mid \mathbf{A}_{k-1} \mathbf{x}_{k-1, j}, \mathbf{Q}_{k-1}\right),
$$

where $\mathbf{A}_{k-1}$ is the time dependent transition matrix and $\mathbf{Q}_{k-1}$ is the time dependent process noise covariance matrix. The dynamics of different targets are assumed to be independent. The measurement model is such that each measurement corresponds to a randomly selected target, denoted by $c_{k}$ and conditional on the association, the measurement depends only on the state of target $c_{k}$. In particular, the measurements conditional on target states and associations are linear Gaussian:

$$
p\left(\mathbf{y}_{k} \mid \mathbf{x}_{k, j}, c_{k}=j\right)=\mathcal{N}\left(\mathbf{y}_{k} \mid \mathbf{H}_{k} \mathbf{x}_{k, j}, \mathbf{R}_{k}\right),
$$

where $\mathbf{H}_{k}$ is the measurement matrix and $\mathbf{R}_{k}$ is the measurement noise covariance matrix.

Unknown and varying number of targets is handled by defining an indicator variable $\mathbf{e}_{k}$ which tells which of the targets are alive at the current time step. The initial state has no targets and the targets are assumed to enter the state at the time of their first observation. Targets are removed 
from consideration by setting the indicator to 0 after a target has not been observed for a while. Särkkä et al. (2007) also consider removing targets probabilistically based on time since last observation. Since the targets are labeled according to the order they are first observed, the data association prior $p\left(c_{k} \mid c_{k-1}, \ldots, c_{1}, \mathbf{e}_{k-1}\right)$ contains positive probabilities only for the targets contained in $c_{1}, \ldots, c_{k-1}$ that are visible in $\mathbf{e}_{k-1}$ as well as one new target. Clutter measurements, that is, measurements that are not related to any target, are modeled by specifying that $p\left(\mathbf{y}_{k} \mid c_{k}=0, \mathbf{x}_{k,:}\right)$ is some fixed distribution independent of the target states. The state of a new target at the time of its first observation is assumed to follow $\mathcal{N}\left(\mathbf{m}_{0}, \mathbf{P}_{0}\right)$. The resulting RBMCDA filter is shown in pseudocode in Algorithm 2.

The model defined above is of the conditionally linear-Gaussian form (6) so that the latent variable $\mathbf{u}_{k}$ consists of the data association $c_{k}$ and the visibility indicator $\mathbf{e}_{k}$. Thus, a RBPF may be applied. Furthermore, since the state-space of possible data associations is finite, the optimal importance distribution may be used for sampling the data association $c_{k}$.

In practice, a computational speedup may be obtained by performing the Kalman filter prediction and updates need only for each unique data association history instead of all particles, some of which are identical. For simplicity of the presentation, this speedup is not explicitly written out in Algorithm 2,

The algorithm state consists of $N$ particles that represent an approximation of the posterior distribution over data association histories at step $k$. The following information is stored for each particle $i \in\{1, \ldots, N\}$ :

$$
\text { Particle }_{i}=\left(c_{1: k}^{(i)}, \mathbf{m}_{k, 1}^{(i)}, \mathbf{m}_{k, 2}^{(i)}, \ldots, \mathbf{m}_{k, T_{k}^{(i)}}, \mathbf{P}_{k, 1}^{(i)}, \mathbf{P}_{k, 2}^{(i)}, \ldots, \mathbf{P}_{k, T_{k}^{(i)}}, w_{k}^{(i)}\right)
$$


where

- $c_{1: k}^{(i)}$ is the data association history for measurements $1, \ldots, k$

- $T_{k}^{(i)}$ is the number of different targets seen so far, i.e., maximum of $c_{1: k}^{(i)}$

- $\mathbf{m}_{k, j}^{(i)}, \mathbf{P}_{k, j}^{(i)}$ are the mean and covariance of the distribution of the state of target $j$ conditional on $c_{1: k}^{(i)}$

- $w_{k}^{(i)}$ is the importance weight of the particle.

The algorithm proceeds through the measurements as follows:

1. For all particles $i \in\{1, \ldots, N\}$ :

(a) For all targets $j \in\left\{1, \ldots, T_{k}^{(i)}\right\}$, propagate the target state distribution moments through the Kalman filter prediction step to obtain the moments $\mathbf{m}_{k, j}^{(i)-}, \mathbf{P}_{k, j}^{(i)-}$ of the distributions $p\left(\mathbf{x}_{k, j} \mid \mathbf{y}_{1: k-1}, c_{1: k-1}^{(i)}\right)$.

$$
\mathbf{m}_{k, j}^{(i)-}=\mathbf{A}_{k-1} \mathbf{m}_{k-1, j}^{(i)}, \mathbf{P}_{k, j}^{(i)-}=\mathbf{A}_{k-1} \mathbf{P}_{k-1, j} \mathbf{A}_{k-1}^{\top}+\mathbf{Q}_{k-1}
$$

(b) For all targets $j \in\left\{1, \ldots, T_{k}^{(i)}\right\}$, run the Kalman filter update step conditional on the data association to obtain the moments $\mathbf{m}_{k, j}^{(i) *}, \mathbf{P}_{k, j}^{(i) *}$ of the distributions $p\left(\mathbf{x}_{k, j} \mid \mathbf{y}_{1: k}, c_{1: k-1}^{(i)}, c_{k}=j\right)$ and the likelihoods $p\left(\mathbf{y}_{k} \mid c_{1: k}^{(i)}, \mathbf{y}_{1: k-1}\right)$ (See Algorithm 3).

(c) Evaluate the optimal importance distribution

$$
P\left(c_{k}=j\right)=\frac{p\left(c_{k}=j \mid c_{1: k}^{(i)}\right) p\left(\mathbf{y}_{k} \mid c_{1: k}^{(i)}, \mathbf{y}_{1: k-1}\right)}{\sum_{j=1}^{T_{k}^{(i)}+1} p\left(c_{k}=j \mid c_{1: k}^{(i)}\right) p\left(\mathbf{y}_{k} \mid c_{1: k}^{(i)}, \mathbf{y}_{1: k-1}\right)}
$$

(d) Draw $c_{k}^{(i)}$ from the optimal importance distribution

(e) $\operatorname{Set}\left(\mathbf{m}_{k, c_{k}}^{(i)}, \mathbf{P}_{k, c_{k}}^{(i)}\right)=\left(\mathbf{m}_{k, c_{k}}^{(i) *}, \mathbf{P}_{k, c_{k}}^{(i) *}\right)$, 
(f) For $j \neq c_{k}$ : set $\left(\mathbf{m}_{k, j}^{(i)}, \mathbf{P}_{k, j}^{(i)}\right)=\left(\mathbf{m}_{k, j}^{(i)-}, \mathbf{P}_{k, j}^{(i)-}\right)$, the predicted distributions

(g) Update particle weight: $w_{k}^{(i)}:=w_{k-1}^{(i)} \sum_{j=1}^{T_{k}^{(i)}+1} p\left(c_{k}=j \mid c_{1: k}^{(i)}\right) p\left(\mathbf{y}_{k} \mid\right.$ $\left.c_{1: k}^{(i)}, \mathbf{y}_{1: k-1}\right)$

2. Normalize particle weights to sum to unity

3. Possible resampling step

The marginal likelihood approximation similar to Eq. 4 in Section 2.1 is computed by

$$
\hat{p}\left(\mathbf{y}_{1: T} \mid \boldsymbol{\theta}\right)=\prod_{k=1}^{T} \hat{p}\left(\mathbf{y}_{k} \mid \mathbf{y}_{1: k-1}, \boldsymbol{\theta}\right)
$$

where

$$
\hat{p}\left(\mathbf{y}_{k} \mid \mathbf{y}_{1: k-1}, \boldsymbol{\theta}\right)=\sum_{i=1}^{N} k w_{k-1}^{(i)}\left(\sum_{j=1}^{T_{k}^{(i)}+1} p\left(c_{k}=j \mid c_{1: k}^{(i)}\right) p\left(\mathbf{y}_{k} \mid c_{1: k}^{(i)}, \mathbf{y}_{1: k-1}\right)\right) .
$$

\section{PMCMC for RBMCDA}

In this section, we show how the RBMDCA algorithm described in Section 3 can be combined with the PMCMC algorithms described in Section 2 . The model is assumed to be of the linear-Gaussian form specified in Section 3 with the extension that the dynamic model transition matrices $\mathbf{A}_{k}(\boldsymbol{\theta})$, process noise covariances $\mathbf{Q}_{k}(\boldsymbol{\theta})$, measurement model matrices $\mathbf{H}_{k}(\boldsymbol{\theta})$ and measurement noise covariance matrices $\mathbf{R}_{k}(\boldsymbol{\theta})$ depend on some parameter vector $\boldsymbol{\theta}$ of fixed dimension.

The particle marginal Metropolis-Hastings is based on using the particle filter based likelihood approximation. In the RBMCDA context, the PMMH 
algorithm produces a Markov chain moving in the joint space of the parameters and particle sets of the data associations, that is, the samples are of the form $\left(\boldsymbol{\theta}^{j}, c_{1: T}^{j,(1: N)}, w_{T}^{j,(1: N)}\right)$. The algorithm iterates the following at steps $j=1,2, \ldots$ :

1. Draw proposed parameters: $\boldsymbol{\theta}^{*} \sim q\left(\boldsymbol{\theta}^{*} \mid \boldsymbol{\theta}^{j-1}\right)$

2. Run the RBMCDA filter (see Section 3 or Algorithm 2) using the parameters $\boldsymbol{\theta}^{*}$ to obtain weighted set of particles $\left(w_{T}^{*,(1: N)}, c_{1: T}^{*,(1: N)}\right)$ and a marginal likelihood estimate $\hat{p}\left(\mathbf{y}_{1: T} \mid \boldsymbol{\theta}^{*}\right)$

3. With probability

$$
\alpha_{j}=\min \left(1, \frac{q\left(\boldsymbol{\theta}^{j-1} \mid \boldsymbol{\theta}^{*}\right)}{q\left(\boldsymbol{\theta}^{*} \mid \boldsymbol{\theta}^{j-1}\right)} \frac{\hat{p}\left(\mathbf{y}_{1: T} \mid \boldsymbol{\theta}^{*}\right)}{\hat{p}\left(\mathbf{y}_{1: T} \mid \boldsymbol{\theta}^{j-1}\right)} \frac{p\left(\boldsymbol{\theta}^{*}\right)}{p\left(\boldsymbol{\theta}^{j-1}\right)}\right)
$$

accept the proposal, that is:

$$
\begin{aligned}
& \left(\boldsymbol{\theta}^{j}, w_{T}^{j,(1: N)}, c_{1: T}^{j,(1: N)}, \hat{p}\left(\mathbf{y}_{1: T} \mid \boldsymbol{\theta}^{j}\right)\right) \\
:= & \left(\boldsymbol{\theta}^{*}, w_{T}^{*,(1: N)}, c_{1: T}^{*,(1: N)}, \hat{p}\left(\mathbf{y}_{1: T} \mid \boldsymbol{\theta}^{*}\right)\right) .
\end{aligned}
$$

4. If the proposal is not accepted, copy the values from previous iteration:

$$
\begin{aligned}
& \left(\boldsymbol{\theta}^{j}, w_{T}^{j,(1: N)}, c_{1: T}^{j,(1: N)}, \hat{p}\left(\mathbf{y}_{1: T} \mid \boldsymbol{\theta}^{j}\right)\right) \\
:= & \left(\boldsymbol{\theta}^{j-1}, w_{T}^{j-1,(1: N)}, c_{1: T}^{j-1,(1: N)}, \hat{p}\left(\mathbf{y}_{1: T} \mid \boldsymbol{\theta}^{j-1}\right)\right) .
\end{aligned}
$$

Samples from the posterior of data associations are obtained by drawing from $\left(c_{1: T}^{j,(1)}, c_{1: T}^{j,(2)}, \ldots, c_{1: T}^{j,(N)}\right)$ with probabilities $w_{T}^{j,(1: N)}$. In this work, we use symmetric multivariate Gaussian random-walk proposals for parameters. The covariance of the proposal distribution is adapted using the sample covariance of the samples produced so far, following the idea of Haario et al. (2001). 
We adapt the covariance only during initial warmup to ensure the ergodicity of the adapting process is maintained in particle MCMC. The resulting RBMCDA-PMMH algorithm is shown in pseudocode in Algorithm 5.

Following the idea of conditional SMC, also the RBMCDA algorithm can be modified so that one particle is fixed to a given data association history. This also results in a MCMC move whose invariant distribution is the conditional posterior of data associations given parameters. This conditional RBMCDA algorithm is shown in pseudocode in Algorithm 6. Since in general models, the conditional posterior of parameters conditional on the data associations may not be available in closed-form, we replace the Gibbs step of PGibbs by Metropolis-Hastings steps for parameters. Thus, RBMCDAPGibbs algorithm iterates the following steps:

1. Propose new $\boldsymbol{\theta}^{*} \sim q\left(\boldsymbol{\theta}^{*} \mid \boldsymbol{\theta}^{j-1}\right)$

2. With probability

$$
\alpha_{j}=\min \left(1, \frac{q\left(\boldsymbol{\theta}^{j-1} \mid \boldsymbol{\theta}^{*}\right)}{q\left(\boldsymbol{\theta}^{*} \mid \boldsymbol{\theta}^{j-1}\right)} \frac{p\left(\mathbf{y}_{1: T} \mid \boldsymbol{\theta}^{*}, c_{1: T}^{j-1}\right)}{p\left(\mathbf{y}_{1: T} \mid \boldsymbol{\theta}^{j-1}, c_{1: T}^{j-1}\right)} \frac{p\left(\boldsymbol{\theta}^{*}\right)}{p\left(\boldsymbol{\theta}^{j-1}\right)}\right)
$$

accept the proposal, that is, set $\boldsymbol{\theta}^{j}:=\boldsymbol{\theta}^{*}$. Else, set $\boldsymbol{\theta}^{j}:=\boldsymbol{\theta}^{j-1}$.

3. Generate $\left(c_{1: T}^{j,(1: N)}, w_{1: T}^{j,(1: N)}\right)$ by running the conditional RBMCDA (Algorithm 6 using parameters $\boldsymbol{\theta}^{j}$ and fixing the data associations in the first particle to $c_{1: T}^{j-1}$.

4. Sample a data association sequence $c_{1: T}^{j}$ from $c_{1: T}^{j,(1: N)}$ with probabilities $w_{T}^{j,(1: N)}$.

To evaluate the acceptance ratios, the likelihood conditional on data associations, $p\left(\mathbf{y}_{1: T} \mid \boldsymbol{\theta}, c_{1: T}^{j-1}\right)$, needs to be evaluated using the Kalman filter as 
shown in Algorithm 7. For the Metropolis-Hastings proposal distributions $q$, we use the multivariate Gaussian random walk proposal adapted similarly as in the RBMCDA-PMMH algorithm. The resulting RBMCDA-PGibbs algorithm is shown in pseudocode in Algorithm 8 .

In some preliminary experiments, we observed that the conditional RBMCDA move sometimes led to poor mixing as the targets associated to early measurements usually did not change. To improve mixing, we also introduced additional Gibbs sampling steps where the targets associated to some particular measurements are redrawn from their conditional distributions.

\section{Experimental Results}

\subsection{Simulated Data}

In this section we compare the performance of the RBMCDA-PGibbs algorithms with varying number of particles. We generate a simulated dataset and run different MCMC algorithms to estimate the posterior distribution of parameters and data associations. We look at the convergence of the distribution of the number of targets in terms of Kolmogorov distance to a distribution obtained by a longer RBMCDA-PGibbs run. The Kolmogorov distance is compared against the total number of Kalman filter predict and update function calls.

We simulated 30 two-dimensional target trajectories using the OrnsteinUhlenbeck mean-reverting model:

$$
\mathrm{d} \mathbf{x}=\lambda\left(\mathbf{x}_{0}-\mathbf{x}\right) \mathrm{d} t+\sqrt{q} \mathrm{~d} \mathbf{W}
$$

where $\mathbf{x}$ is the target location and $\mathbf{x}_{0}$ is a fixed mean location of the target. The parameters were set to $\lambda=0.5, \sqrt{q}=10$. The mean locations were 
sampled uniformly randomly in the window $[0,100] \times[0,100]$. Initial target locations were drawn from the steady-state distribution of the OrnsteinUhlenbeck process. Then, 150 observation times were sampled uniformly randomly in $[0,1]$, and data associations were generated so that the target associated to each measurement was selected randomly, but the dataassociations were resampled until an association history where every target is obtained at least once was obtained. The measurements were the locations plus uncorrelated Gaussian noise with standard deviation $\sigma=0.5$ in both coordinates. The simulated target movements and observations are shown in Figure 1 .

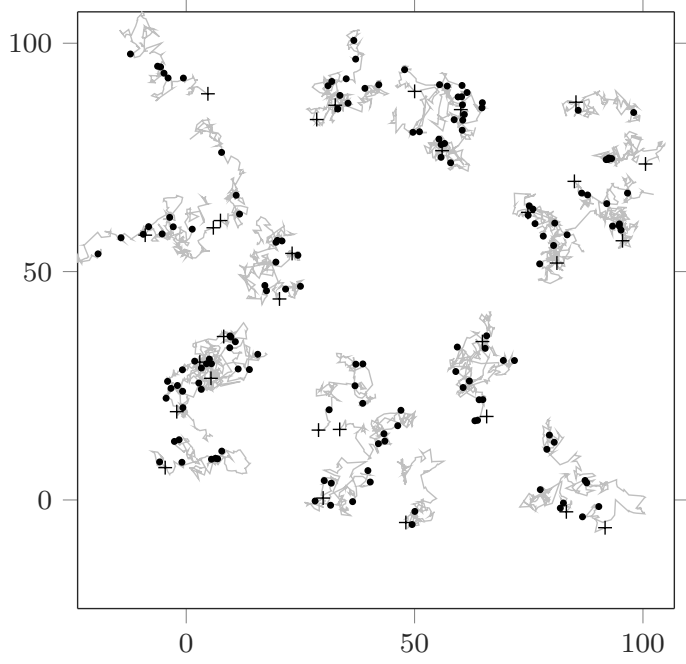

Figure 1: Visualization of the simulated scenario. Trajectories of the targets are shown as gray lines, measurements as black dots and final target locations as black pluses.

10 chains of RBMCDA-PGibbs were run for $10^{6}$ steps. First half was discarded as warmup and the remaining samples from all chains pooled. The Ornstein-Uhlenbeck model was used for the target dynamics so that the tar- 
get state is 4-dimensional consisting of the (constant) mean location and the actual location. The initial density of new targets, $\mathcal{N}\left(\mathbf{m}_{0}, \mathbf{P}_{0}\right)$, was obtained by taking the sample mean and sample covariance of all observations, and using these for the distribution of the mean location coordinates. For the actual location coordinates, the corresponding steady-state distribution was used. Note that the initial density thus depends on the model parameters. For the model parameters we used Gamma priors with scale 2 (Chung et al., 2013) and modes $(\sqrt{q}=15, \lambda=1 / 3, \sigma=0.75)$. These modes were selected so that the prior mode is somewhat off from the ground truth and favors a smaller number of targets. The data association prior $p\left(c_{k} \mid c_{1: k-1}\right)$ was obtained as follows. The probability of new target is set to the conditional probability of a new target appearing conditional on a latent number of targets drawn uniformly from $\{1, \ldots$, number of observations $\}$ and each association being drawn uniformly from the latent number of targets. All old targets have equal probability. No clutter measurements nor target deaths were used.

We checked MCMC convergence using the potential scale reduction factor Gelman et al. 2013) with the implementation in GPStuff (Vanhatalo et al., 2013). Using the latter halves of the 10 chains, the PSRF for all 3 static parameters was below 1.01, so we conclude that the chains have converged and pooling samples from the different chains is justified. The results are shown in Figure 2. The number of targets is slightly underestimated, which is natural as the parameter prior modes was set to favor a smaller number of targets compared to the true parameters. The posteriors of $\sqrt{q}$ and the measurement error $\sigma$ are clearly thinner than the prior and the modes are moved towards the truth. The posterior of the mean-reversion rate $\lambda$ is rather 
Table 1: The simulated experiment. Comparison of RBMCDA-PGibbs with and without parameter estimation. Posterior probability of 30 targets (the ground truth) as well as mean OSPA metric of the final target locations.

\begin{tabular}{c|cc} 
Parameter estimation & $\mathrm{P}$ (Correct number of targets) & Mean OSPA \\
\hline Yes & 0.14 & 5.95 \\
No & 0.005 & 8.02
\end{tabular}

wide. This is explained by the fact that the time window of the simulation was quite short relative to the value of $\lambda$. However, the posterior of $\lambda$, too, was slightly moved towards true value.

To investigate the usefulness of parameter estimation, we also ran the RBMCDA-PGibbs with the same number of particles and chain lengths without sampling for parameters, that is, using the initial parameter values. We compared the accuracy based on the probability of the true number of targets as well as the OSPA metric (Schuhmacher et al., 2008) for the posterior mean locations for all targets at the time of the 150th measurement. The results are shown in Table 1. To save computational resources, the OSPA metric was computed using only every 500th step of the MCMC chains.

We tried RBMCDA-PGibbs with 5 and 100 particles both with and without additional Gibbs steps. For each algorithm, 5 independent chains were used. Figure 3 shows Kolmogorov distances to the distribution of Figure 2 as a function of Kalman filter function evaluations. These are evaluated by cutting the chains at selected sample sizes, pooling results from all 5 chains 
and discarding first half as warmup!
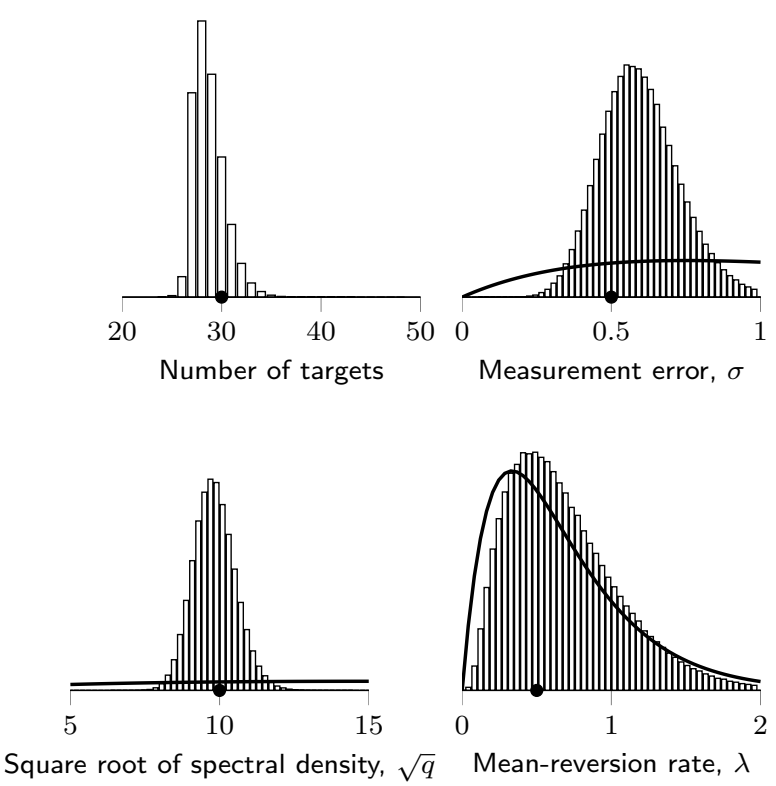

Figure 2: Posterior distributions of the parameters $(\sqrt{q}, \sigma, \lambda)$ and the number of targets in the simulated scenario. The corresponding prior densities for parameters are shown as solid lines. Ground-truth parameters are marked as dots on the axis.

\subsection{Real Data: Estimating Brown Bear Population}

We consider a dataset of location records of direct sightings and fieldsign observations of brown bears in Finland provided by Finnish Game and Fisheries Research institute. The main quantity of interest in this study is the number of distinct packs (families) observed, which can then be used to

\footnotetext{
${ }^{1}$ To save computation time, the chains used for RBMCDA-PGibbs with Gibbs steps and 5 particles are 5 first chains of the 10 that were used to produce the ground truth. However, all samples used to this plot are discarded as warmup in the gold-standard distribution, so this is unlikely to bias the results.
} 


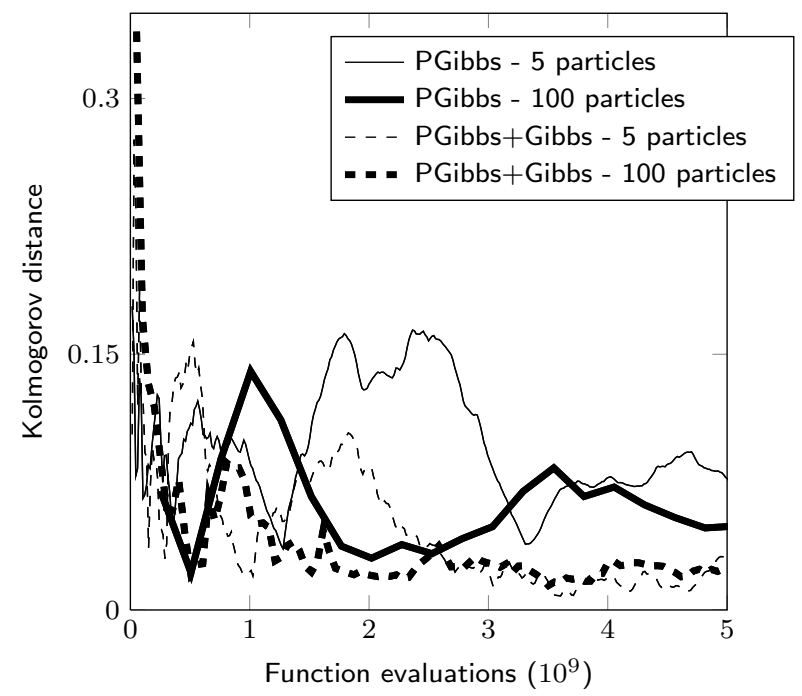

Figure 3: Convergence of the distribution of the number of targets with varying algorithms. Kolmogorov distance vs. number of Kalman filter function evaluations.

estimate the overall population size by using an extrapolation factor $\mathrm{Ko}-$ jola, 2007). We use a probabilistic approach for estimating the number of distinct families by formulating the problem as a multiple target tracking problem, where the targets are the packs. The posterior distribution for the number of packs is then obtained as a byproduct of the multiple target tracking solution. We used data of observations from year 2013 selecting only observations where cubs were present. We selected observations from one game management district (Kaakkois-Suomen riistanhoitopiiri). Abbas (2011) used RBMCDA in his Master's thesis for population estimation with this type of data, but this work did not use PMCMC for parameter estimation.

For target movement, we used the Ornstein-Uhlenbeck mean-reverting model (cf. Section 5), and measurement locations were assumed to be the 
actual target location plus Gaussian noise independent in both coordinates. Conditional on the parameters, the target dynamics of each year was assumed to be independent. Weakly informative $\operatorname{Gamma}(2, \mu)$-priors were used for the parameters with modes: $\sqrt{q}=2500 \mathrm{~m} / \mathrm{d}, \lambda=0.5 \mathrm{~d}^{-1}, \sigma=100 \mathrm{~m}$. We used 5 particles and 10 separate MCMC chains were run for 100,000 steps each. The results presented here are based on discarding the first half of each chain as warmup and combining the remaining samples from all 10 chains.

Histograms of the posterior distributions of parameter and number of targets compared to prior densities are shown in Figure 4. Compared to the expert estimates by Finnish Game and Fisheries Research institute (FGFRI, 2014), the model clearly overestimates the number of packs - the expert estimate was $20-22$ while our model predicts about $60-80$ targets. However, this may be due to experts having more information about, for example, which observations are unreliable. Furthermore, it may be that our prior distributions were too noninformative, placing considerable mass on unrealistic parameter values. Indeed, the posterior for the parameter $q$ in the posterior is much smaller than the prior expectation, which naturally explains the high number of targets.

\section{Conclusions and Discussion}

In this paper, we have presented a novel algorithm for parameter estimation in multiple target tracking problems. The algorithm is based on combining the Rao-Blackwellized Monte Carlo data association (RBMCDA) algorithm (Särkkä et al., 2007) with particle Markov chain Monte Carlo (PMCMC) methods (Andrieu et al., 2010). We considered two different varia- 

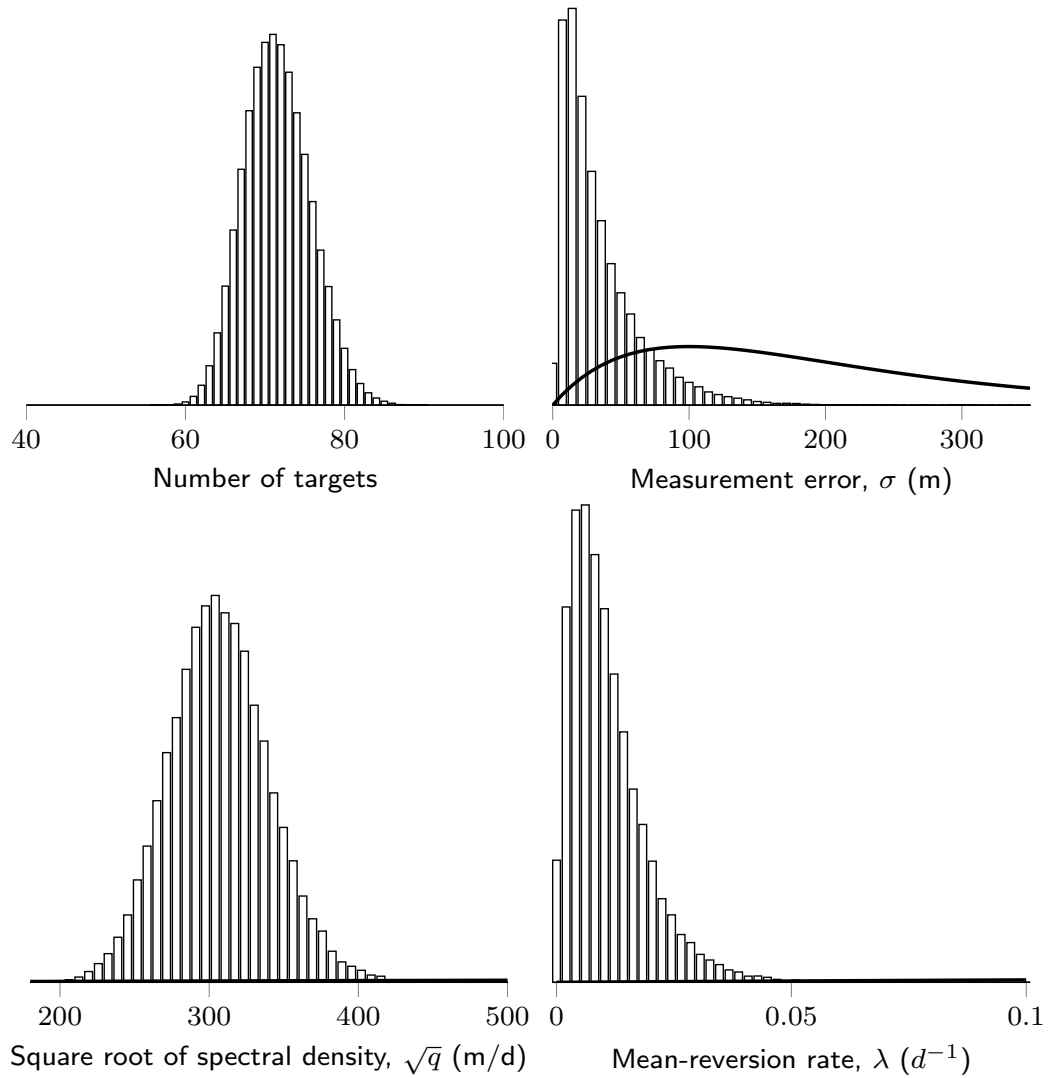

Figure 4: Posterior distributions of the number of brown bear families in the KaakkoisSuomi district in year 2013, and the model parameters. Solid lines denote the corresponding prior densities.

tions of the algorithm based on the particle marginal Metropolis-Hastings and particle Gibbs algorithms known in the particle MCMC literature.

In the numeric experiments section, we tested the method with a simulated example and then applied it to a real-data application of estimating the brown bear population in Finland. With the simulated data, we also compared the convergence of the distribution of targets with different variations of our algorithm. 
This research could be continued in several directions. To speed up computations, one could combine gating techniques with RBMCDA (Wang and Zhang, 2014). It may be possible to derive an upper bound for the measurement likelihoods such that early rejection (Solonen et al., 2012) could be applied in RBMCDA-PMMH. That is, computational speedup would be obtained by sometimes deducing during a RBMCDA step that a proposal will be rejected, without processing through all measurements. Besides sampling the parameters of the dynamic and measurement models, one could sample the data association priors as well as the initial densities. Särkkä et al. (2007) showed that the RBMCDA algorithm can be easily extended to nonlinear models by using an approximative filter, such as the EKF or the UKF (Särkkä, 2013). This extension could as well be combined with PMCMC. Rao-Blackwellized particle smoothing (Särkkä et al., 2012; Lindsten et al., 2013) could be used to obtain smoothing distributions of the target states. The model could be extended to allow separate parameters for each target. With unknown number of targets, this would require reversible jump MCMC (Green, 1995; Punskaya et al., 2002) or similar techniques. Allowing interaction among target states would enable group tracking (see Mihaylova et al., 2014, and references therein). Besides particle MCMC, one could investigate other methods combining particle filters with inference on static parameters, such as $\mathrm{SMC}^{2}$ (Chopin et al., 2013) and particle learning (Carvalho et al. 2010), in the RBMCDA context. 


\section{Acknowledgments}

This work was supported by grants from the Academy of Finland (266940, 273475). We acknowledge the computational resources provided by the Aalto Science-IT project. We thank Samuli Heikkinen and Mika Kurkilahti from the Finnish Game and Fisheries Research institute for providing the real data and helpful discussions. We thank Arno Solin, Joona Karjalainen, and three anonymous reviewers for helpful comments on the manuscript.

\section{References}

\section{References}

Abbas, M., 2011. Statistical estimation of wild animal population in Finland: a multiple target tracking approach. Master's thesis, School of Science, Aalto University, Finland.

Akashi, H., Kumamoto, H., 1977. Random sampling approach to state estimation in switching environments. Automatica 13 (4), 429-434.

Andrieu, C., Doucet, A., Holenstein, R., 2010. Particle Markov chain Monte Carlo methods. Journal of the Royal Statistical Society: Series B (Statistical Methodology) 72 (3), 269-342.

Andrieu, C., Doucet, A., Singh, S., Tadic, V., March 2004. Particle methods for change detection, system identification, and control. Proceedings of the IEEE 92 (3), 423-438.

Bar-Shalom, Y., Li, X., Li, X., Kirubarajan, T., 2001. Estimation with Applications to Tracking and Navigation. Wiley-Interscience. 
Blackman, S., Popoli, R., 1999. Design and Analysis of Modern Tracking Systems. Artech House Norwood, MA.

Blackman, S. S., 2004. Multiple hypothesis tracking for multiple target tracking. Aerospace and Electronic Systems Magazine, IEEE 19 (1), 5-18.

Bugallo, M. F., Lu, T., Djuric, P. M., 2007. Target tracking by multiple particle filtering. In: Aerospace Conference, 2007 IEEE. IEEE, pp. 1-7.

Carvalho, C., Johannes, M. S., Lopes, H. F., Polson, N., 2010. Particle learning and smoothing. Statistical Science 25 (1), 88-106.

Challa, S., Morelande, M., Musicki, D., Evans, R., 2011. Fundamentals of object tracking. Cambridge University Press.

Chen, R., Liu, J. S., 2000. Mixture Kalman filters. Journal of the Royal Statistical Society: Series B (Statistical Methodology) 62 (3), 493-508.

Chopin, N., 2010. Discussion to 'Particle Markov chain Monte Carlo methods' by C. Andrieu, A. Doucet and R. Holenstein. Journal of the Royal Statistical Society: Series B (Statistical Methodology) 72 (3), 304-305.

Chopin, N., Jacob, P. E., Papaspiliopoulos, O., 2013. SMC2: an efficient algorithm for sequential analysis of state space models. Journal of the Royal Statistical Society: Series B (Statistical Methodology) 75 (3), 397426.

Chung, Y., Rabe-Hesketh, S., Dorie, V., Gelman, A., Liu, J., 2013. A nondegenerate penalized likelihood estimator for variance parameters in multilevel models. Psychometrika 78 (4), 685-709. 
Clark, D., Vo, B.-T., Vo, B.-N., 2007. Gaussian particle implementations of probability hypothesis density filters. In: Aerospace Conference, 2007 IEEE. IEEE, pp. 1-11.

Clark, D. E., Bell, J., 2007. Multi-target state estimation and track continuity for the particle PHD filter. Aerospace and Electronic Systems, IEEE Transactions on 43 (4), 1441-1453.

Closas, P., Bugallo, M. F., 2012. Improving accuracy by iterated multiple particle filtering. Signal Processing Letters, IEEE 19 (8), 531-534.

Djuric, P. M., Bugallo, M. F., 2009. Improved target tracking with particle filtering. In: Aerospace conference, 2009 IEEE. IEEE, pp. 1-7.

Doucet, A., De Freitas, N., Murphy, K., Russell, S., 2000a. Rao-Blackwellised particle filtering for dynamic Bayesian networks. In: Proceedings of the Sixteenth conference on Uncertainty in artificial intelligence. Morgan Kaufmann Publishers Inc., pp. 176-183.

Doucet, A., Godsill, S., Andrieu, C., 2000b. On sequential Monte Carlo sampling methods for Bayesian filtering. Statistics and computing 10 (3), 197208.

Duckworth, D., 2012. Monte Carlo methods for multiple target tracking and parameter estimation. Berkeley technical report.

Fearnhead, P., Clifford, P., 2003. On-line inference for hidden Markov models via particle filters. Journal of the Royal Statistical Society: Series B (Statistical Methodology) 65 (4), 887-899. 
Fernández-Villaverde, J., Rubio-Ramírez, J. F., 2007. Estimating macroeconomic models: A likelihood approach. The Review of Economic Studies 74 (4), 1059-1087.

FGFRI, 2014. Population estimates by the finnish game and fisheries research institute (in Finnish). http://www.rktl.fi/riista/suurpedot/ rktln_lausunnot_suurpedoista.html.

Fortmann, T. E., Bar-Shalom, Y., Scheffe, M., 1980. Multi-target tracking using joint probabilistic data association. In: Decision and Control including the Symposium on Adaptive Processes, 1980 19th IEEE Conference on. Vol. 19. IEEE, pp. 807-812.

Gelman, A., Carlin, J. B., Stern, H. S., Dunson, D. B., Vehtari, A., Rubin, D. B., 2013. Bayesian Data Analysis, 3rd Edition. Chapman \& Hall/CRC.

Green, P. J., 1995. Reversible jump Markov chain Monte Carlo computation and Bayesian model determination. Biometrika 82 (4), 711-732.

Haario, H., Saksman, E., Tamminen, J., 2001. An adaptive Metropolis algorithm. Bernoulli, 223-242.

Hwang, I., Balakrishnan, H., Roy, K., Tomlin, C., 2004. Multiple-target tracking and identity management in clutter, with application to aircraft tracking. In: American Control Conference, 2004. Proceedings of the 2004. Vol. 4. IEEE, pp. 3422-3428.

Jones, E., Parslow, J., Murray, L., 2010. A Bayesian approach to state and parameter estimation in a phytoplankton-zooplankton model. Australian Meteorological and Oceanographic Journal 59, 7-16. 
Kalman, R. E., 1960. A new approach to linear filtering and prediction problems. Journal of Fluids Engineering 82 (1), 35-45.

Kirubarajan, T., Bar-Shalom, Y., 2005. Probabilistic data association techniques for target tracking in clutter. Proceedings of the IEEE 92 (3), 536557.

Kojola, I., 2007. Biology of the bear and the current status of the bear population. In: Management Plan for the Bear Population in Finland. Publications of Ministry of Agriculture and Forestry, Finland, pp. 10-17.

Lindsten, F., Bunch, P., Godsill, S. J., Schon, T. B., 2013. Rao-Blackwellized particle smoothers for mixed linear/nonlinear state-space models. In: Acoustics, Speech and Signal Processing (ICASSP), 2013 IEEE International Conference on. IEEE, pp. 6288-6292.

Liu, J. S., Chen, R., 1995. Blind deconvolution via sequential imputations. Journal of the American Statistical Association 90 (430), 567-576.

Mahler, R. P. S., 2003. Multitarget Bayes filtering via first-order multitarget moments. IEEE Transactions on Aerospace and Electronic Systems 39 (4), $1152-1178$.

Mahler, R. P. S., 2007a. PHD filters of higher order in target number. IEEE Transactions on Aerospace and Electronic Systems 43 (4), 1523-1543.

Mahler, R. P. S., 2007b. Statistical Multisource-Multitarget Information Fusion. Artech House. 
Martino, L., Leisen, F., Corander, J., 2015. On multiple try schemes and the particle Metropolis-Hastings algorithm, viXra preprint: http://www.rxiv.org/pdf/1409.0051v2.pdf.

Mestre, M. R., Fitzgerald, W. J., 2013. Multi-target tracking applied to evolutionary clustering. In: Acoustics, Speech and Signal Processing (ICASSP), 2013 IEEE International Conference on. IEEE, pp. 3173-3177.

Mihaylova, L., Carmi, A. Y., Septier, F., Gning, A., Pang, S. K., Godsill, S., 2014. Overview of Bayesian sequential Monte Carlo methods for group and extended object tracking. Digital Signal Processing 25, 1-16.

Minvielle, P., Todeschini, A., Caron, F., Del Moral, P., 2014. Particle MCMC for Bayesian microwave control. arXiv preprint arXiv:1405.2673.

Nevat, I., Peters, G. W., Yuan, J., 2011. Channel tracking in relay systems via particle MCMC. In: Vehicular Technology Conference (VTC Fall), 2011 IEEE. IEEE, pp. 1-5.

Orton, M., Fitzgerald, W., 2002. A Bayesian approach to tracking multiple targets using sensor arrays and particle filters. Signal Processing, IEEE Transactions on 50 (2), 216-223.

Peters, G., Cornebise, J., 2010. Discussion to 'Particle Markov chain Monte Carlo methods' by C. Andrieu, A. Doucet and R. Holenstein. Journal of the Royal Statistical Society: Series B (Statistical Methodology) 72 (3), 304-305.

Peters, G. W., Briers, M., Shevchenko, P., Doucet, A., 2013. Calibration and filtering for multi factor commodity models with seasonality: incorporating 
panel data from futures contracts. Methodology and Computing in Applied Probability 15 (4), 841-874.

Petetin, Y., Morelande, M., Desbouvries, F., 2014. Marginalized particle PHD filters for multiple object Bayesian filtering. Aerospace and Electronic Systems, IEEE Transactions on 50 (2), 1182-1196.

Punskaya, E., Andrieu, C., Doucet, A., Fitzgerald, W. J., 2002. Bayesian curve fitting using MCMC with applications to signal segmentation. Signal Processing, IEEE Transactions on 50 (3), 747-758.

Rao, G. M., Satyanarayana, C., 2013. Visual object target tracking using particle filter: A survey. International Journal of Image, Graphics and Signal Processing 5 (6), 1250.

Ravindra, V. C., Svensson, L., Hammarstrand, L., Morelande, M., 2012. A cardinality preserving multitarget multi-Bernoulli RFS tracker. In: Information Fusion (FUSION), 2012 15th International Conference on. IEEE, pp. 832-839.

Reid, D. B., 1979. An algorithm for tracking multiple targets. Automatic Control, IEEE Transactions on 24 (6), 843-854.

Särkkä, S., 2013. Bayesian Filtering and Smoothing. Vol. 3 of Institute of Mathematical Statistics Textbooks. Cambridge University Press.

Särkkä, S., Bunch, P., Godsill, S., 2012. A backward-simulation based RaoBlackwellized particle smoother for conditionally linear Gaussian models. In: Proceedings of the 16th IFAC Symposium on System Identification, Brussels, Belgium. 
Särkkä, S., Vehtari, A., Lampinen, J., 2007. Rao-Blackwellized particle filter for multiple target tracking. Information Fusion 8 (1), 2-15.

Schuhmacher, D., Vo, B.-T., Vo, B.-N., 2008. A consistent metric for performance evaluation of multi-object filters. Signal Processing, IEEE Transactions on 56 (8), 3447-3457.

Solonen, A., Ollinaho, P., Laine, M., Haario, H., Tamminen, J., Järvinen, H., et al., 2012. Efficient MCMC for climate model parameter estimation: Parallel adaptive chains and early rejection. Bayesian Analysis 7 (3), 715736.

Svensson, L., Morelande, M., 2014. Target tracking based on estimation of sets of trajectories. In: Information Fusion (FUSION), 2014 17th International Conference on. IEEE, pp. 1-8.

Svensson, L., Svensson, D., Guerriero, M., Willett, P., 2011. Set JPDA filter for multitarget tracking. Signal Processing, IEEE Transactions on 59 (10), 4677-4691.

Vanhatalo, J., Riihimäki, J., Hartikainen, J., Jylänki, P., Tolvanen, V., Vehtari, A., 2013. GPstuff: Bayesian modeling with Gaussian processes. The Journal of Machine Learning Research 14 (1), 1175-1179.

Vihola, M., 2007. Rao-Blackwellised particle filtering in random set multitarget tracking. Aerospace and Electronic Systems, IEEE Transactions on $43(2), 689-705$.

Vo, B.-N., Ma, W.-K., 2006. The Gaussian mixture probability hypothesis density filter. Signal Processing, IEEE Transactions on 54 (11), 4091-4104. 
Vo, B.-N., Singh, S., Doucet, A., 2003. Sequential Monte Carlo implementation of the PHD filter for multi-target tracking. In: Proc. Intl Conf. on Information Fusion. pp. 792-799.

Vo, B.-T., Vo, B.-N., Cantoni, A., 2009. The cardinality balanced multitarget multi-Bernoulli filter and its implementations. Signal Processing, IEEE Transactions on 57 (2), 409-423.

Vu, T., Vo, B.-N., Evans, R., 2014. A particle marginal Metropolis-Hastings multi-target tracker. Signal processing, IEEE Transactions on 62 (15), 3953-3964.

Wang, Y., Zhang, P., 2014. Gating techniques for Rao-Blackwellized Monte Carlo Data Association filter. The Scientific World Journal 2014.

Whiteley, N., Andrieu, C., Doucet, A., 2010. Efficient Bayesian inference for switching state-space models using discrete particle Markov chain Monte Carlo methods. arXiv preprint arXiv:1011.2437.

Yi, W., Morelande, M. R., Kong, L., Yang, J., 2013. A computationally efficient particle filter for multitarget tracking using an independence approximation. Signal Processing, IEEE Transactions on 61 (4), 843-856. 


\section{Appendix A. Algorithms}

In this section, we present the algorithms discussed in the paper in pseudocode.

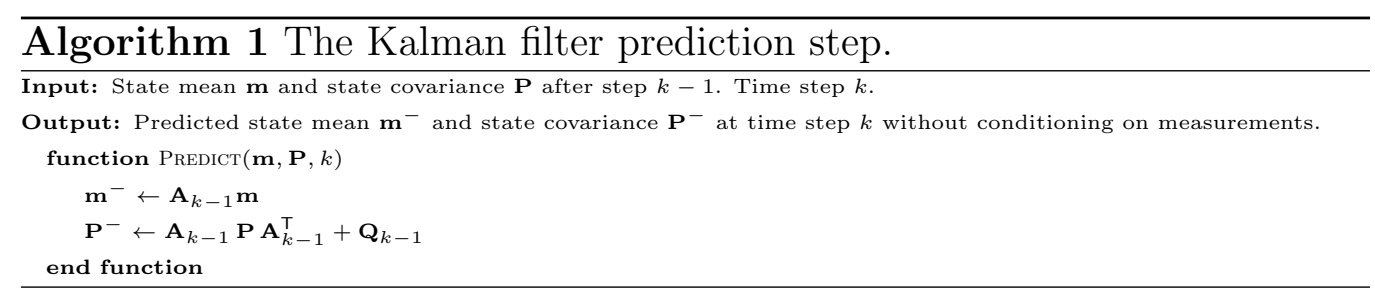


Algorithm 2 The Rao-Blackwellized Monte Carlo data association algo-

rithm.








$\overline{\text { Algorithm } 4 \text { Algorithm for evaluating the unnormalized optimal impor- }}$ tance distribution and updated target states conditional on associations.

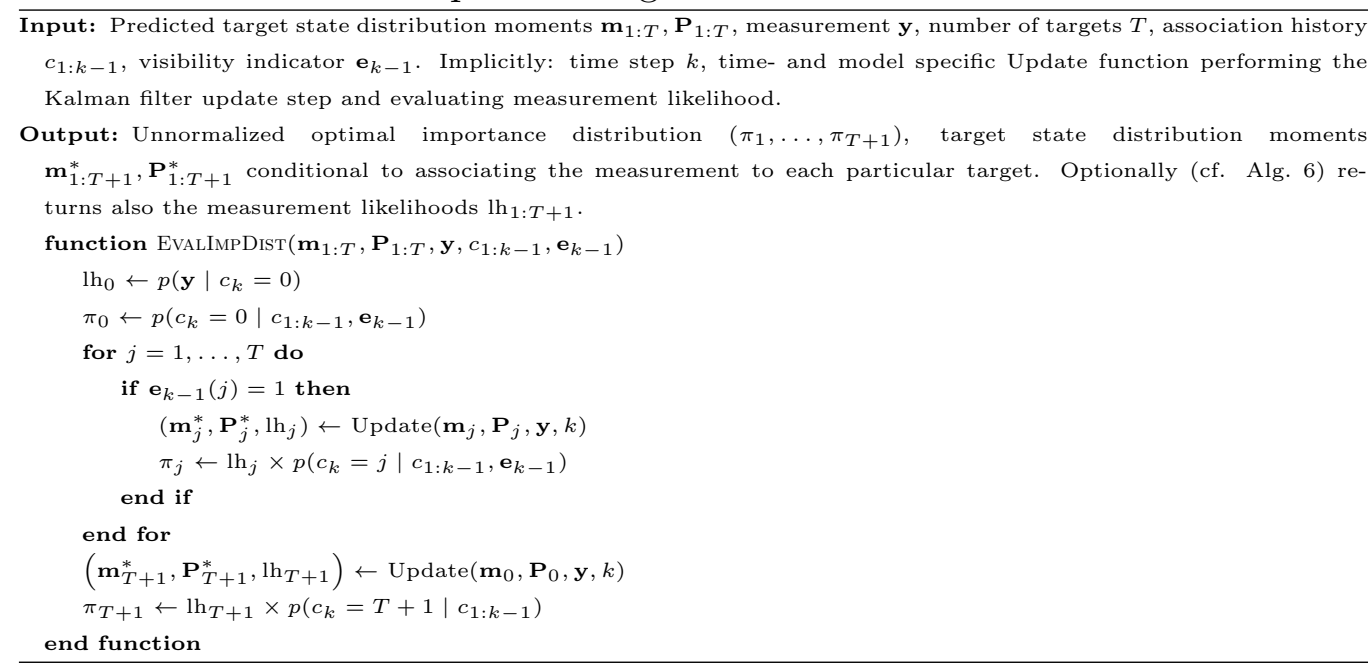




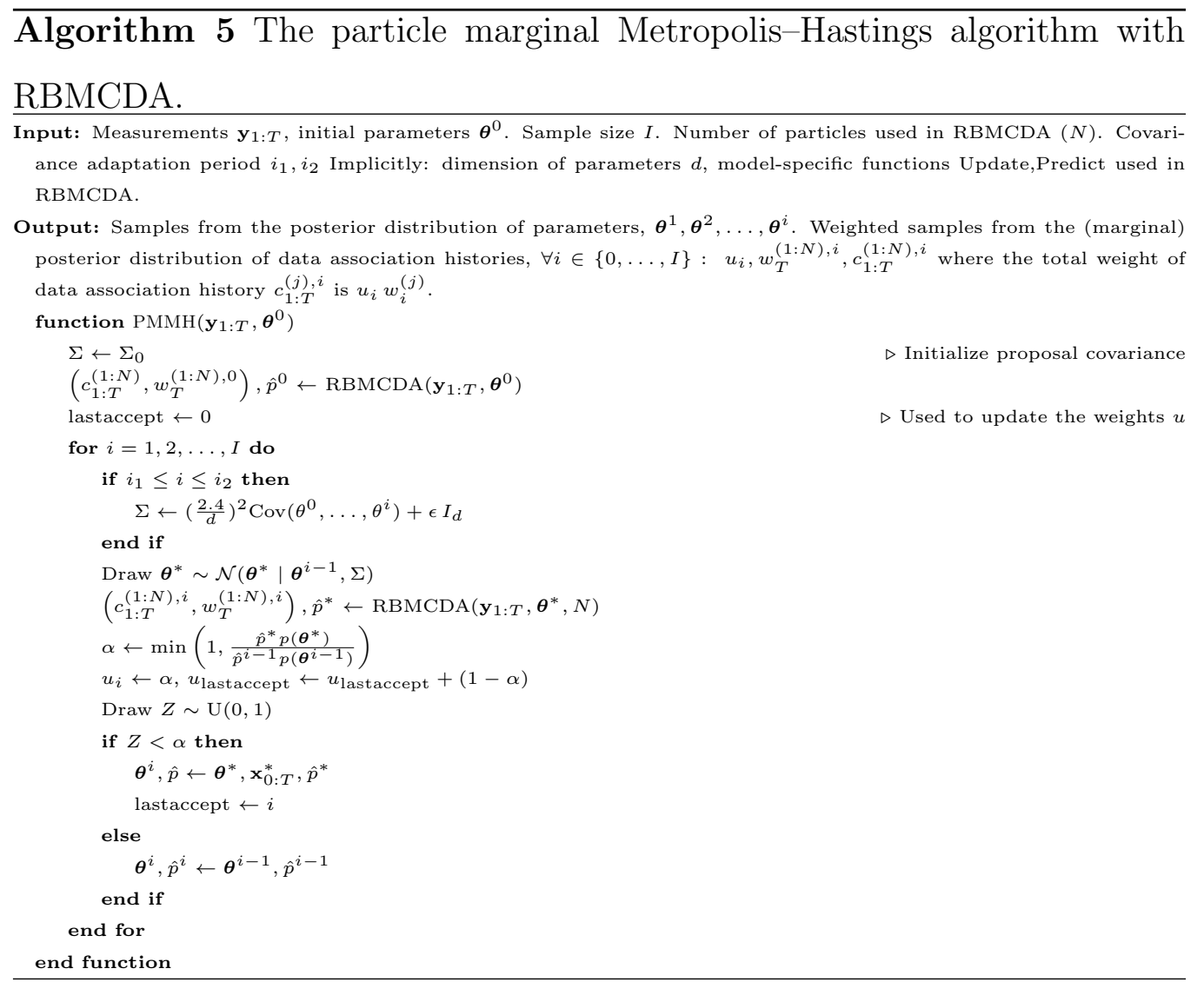




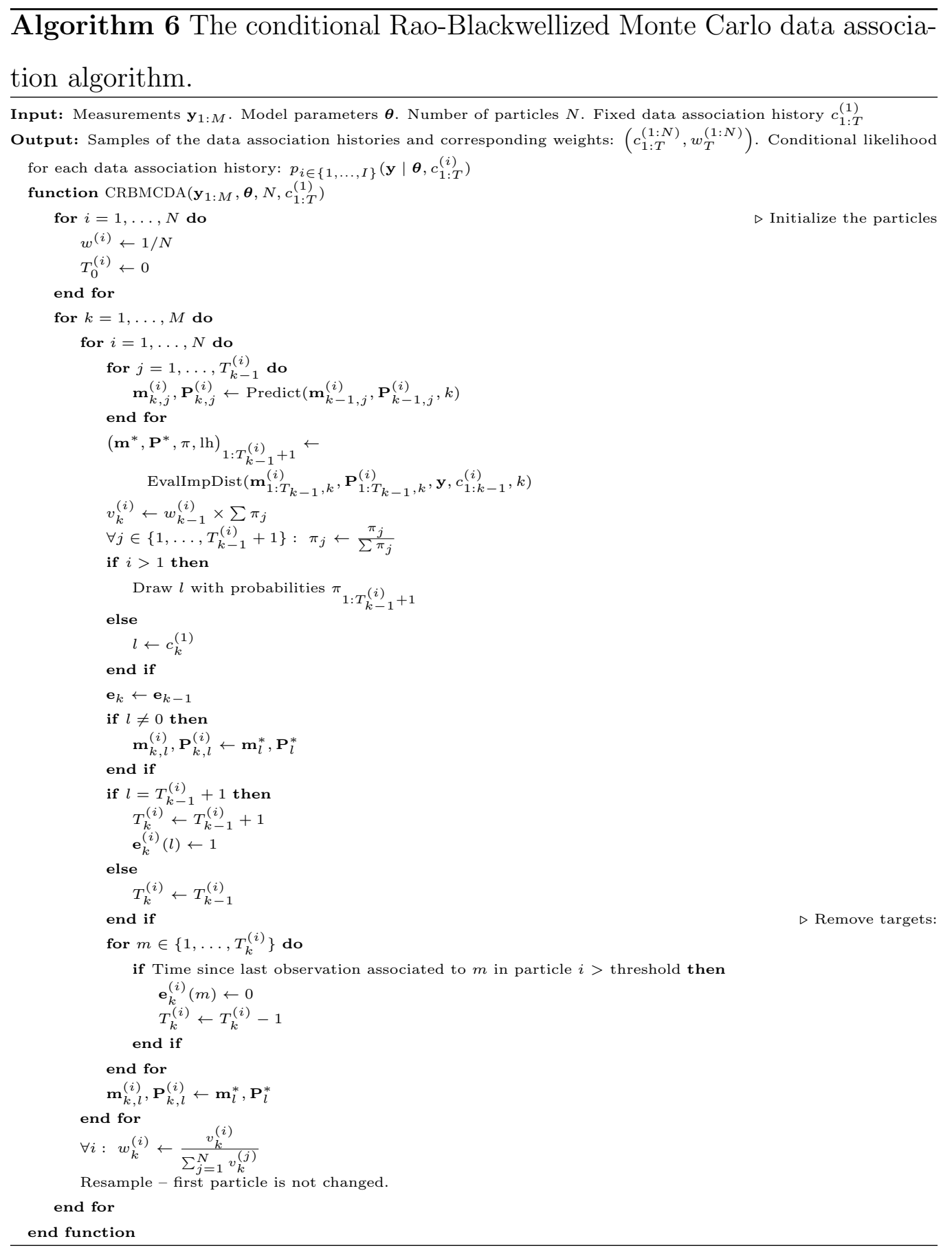




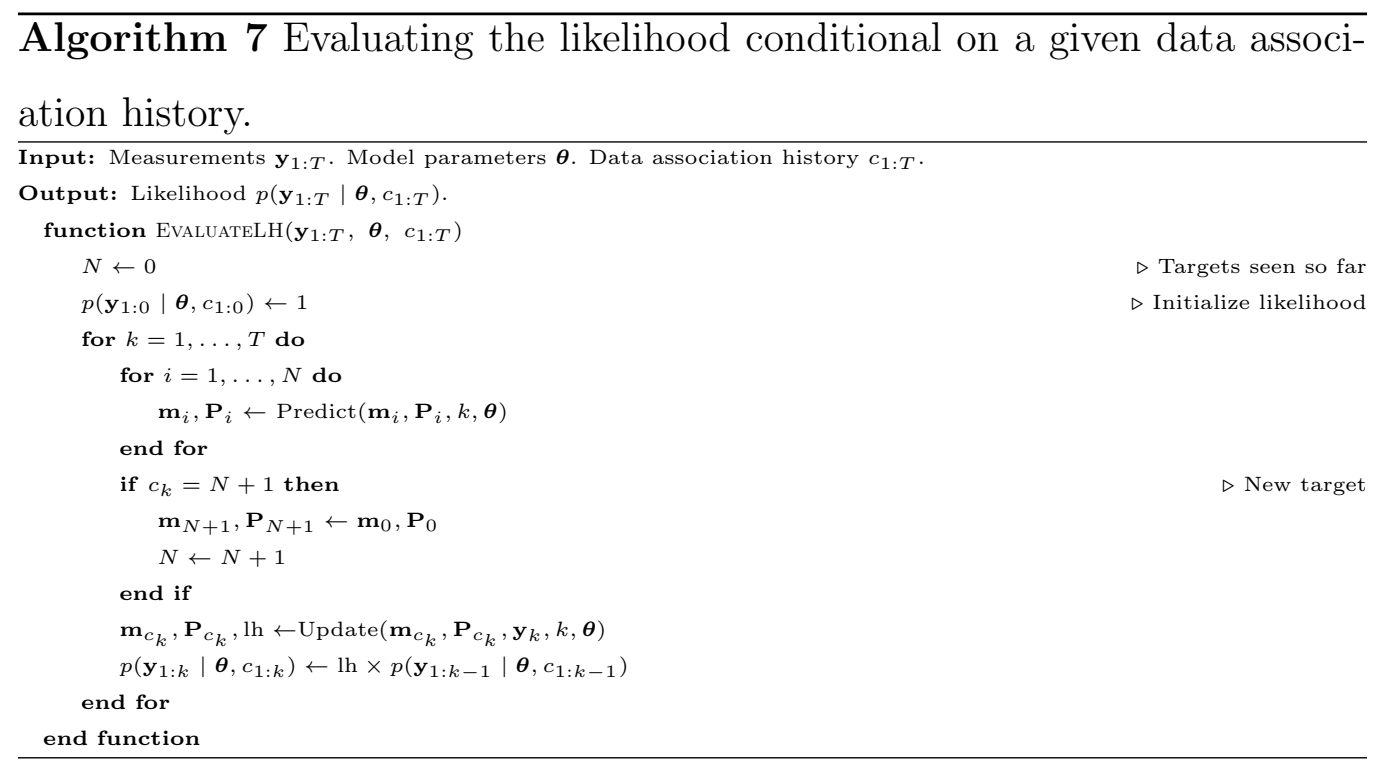




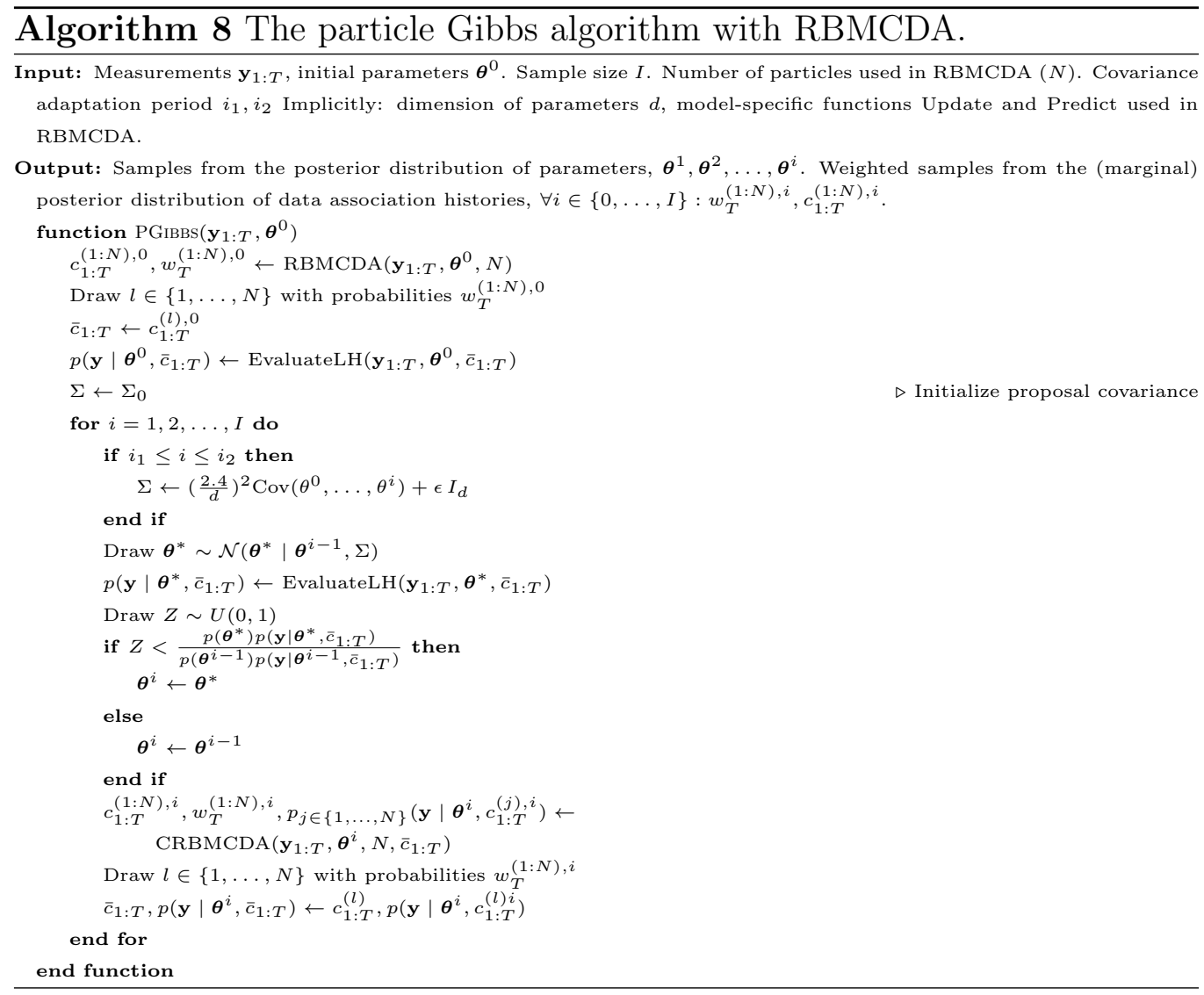

